\title{
Changing Epidemiology, Treatment, and Vaccine Update on Chikungunya, Dengue, and Zika Viruses
}

\author{
Adekunle Sanyaolu ${ }^{1} \cdot$ Oladapo Ayodele $^{2} \cdot$ Lorena Likaj $^{2} \cdot$ Aleksandra Marinkovic $^{2} \cdot$ Jennifer Locke $^{2} \cdot$ Miriam Ahmed $^{2}$. \\ Odunayo Akanbi $^{2} \cdot$ Verner Orish $^{3} \cdot$ Chuku Okorie $^{4}$. Olanrewaju Badaru ${ }^{1}$
}

Published online: 8 May 2019

(C) Springer Nature Switzerland AG 2019

\begin{abstract}
Purpose of Review Now more than ever, regions other than Africa and Asia, such as the USA, are being affected by the rising epidemic of vector-borne illnesses, specifically Chikungunya, Dengue, and Zika viruses; this has prompted this review aimed at discussing the changing epidemiology of the three diseases as well as the current treatment and vaccines in development to control the diseases. With the viruses being spread through a variety of ways, including, but not limited to, mosquito bites, fetal transmission, sexual contact, breast milk, and saliva, there is no doubt that more preventative measures are required.

Recent Findings The changing epidemiology of the three viruses is already creating an impact, with the spread of Dengue in 2009 in Florida, to the 2013 spread of Chikungunya through the Caribbean, and now, the Zika virus making its mark on the tropics with major concerns of it spreading to the Western Hemisphere, including the USA. Although, they are all vector-borne illnesses, each carries its own clinical presentations that sometimes make it hard to diagnose. Collectively, there are no current vaccines or antiviral drugs against these three viruses, and with no sign of the spread slowing down, more geographic regions are in danger of being hit by these diseases in the near future.

Summary As the evolving world for the three viruses continues due to changes in epidemiology, there is a dire need to develop vaccines for each of the three diseases that will target a variety of mechanisms to help fight the transmission and provide succor to affected communities. Public health preventive strategies need to be employed for proper actions to be take aimed at preventing viral transmission and ultimately, helping to fight this changing epidemiology of vector-borne diseases.
\end{abstract}

Keywords Chikungunya virus (CHIKV) · Chikungunya fever (CHIKF) · Dengue fever (DF) · Dengue virus (DENV) $\cdot$ Dengue hemorrhagic fever (DHF) · Dengue shock syndrome (DSS) · Zika virus (ZIKV) · Aedes albopictus · Aedes aegypti

\section{Introduction}

Vector-borne diseases have been on the rise in the past decade, and with no vaccines or appropriate antiviral medications currently available, no region is left safe as the spread

This article is part of the Topical Collection on Tropical Medicine Updates

Adekunle Sanyaolu sanyakunle@gmail.com

1 Federal Ministry of Health, Abuja, Nigeria

2 Saint James School of Medicine, British West Indies, Anguilla

3 University of Health and Allied Sciences, Ho, Volta Region, Ghana

4 Essex County College, Newark, NJ, USA continuously grows, specifically, the Chikungunya, Dengue, and the Zika viruses. All three have similarities that show how one common vector can cause such massive destruction in communities, as well as in the population pool of international travelers. The mosquito Aedes aegypti is known to transmit the Chikungunya, Dengue, and Zika viruses, and with the development of these viruses, these mosquito vectors can continuously spread disease, posing a severe threat to public health worldwide [1,2].

Chikungunya is an arthropod-borne virus of the Togaviridae family transmitted via the Aedes mosquito [3]. Chikungunya virus (CHIKV) in Africa is maintained in a sylvatic cycle among animals, including wild primates, squirrels, birds, and rodents [4]. Those infected with the virus can often present with fever, rash, and severe polyarthralgia, which could possibly lead to a more chronic phase causing death especially in the elderly, neonates, and patients with 
cardiovascular disorders. Patients often complain of loss of function to tarsal joints (hands, fingers, and elbows), making everyday life more complicated [3]. With the spread of the virus through the Caribbean in 2013 and enhanced multiinternational population migration, spreading of the virus is on the rise and has thus far affected areas like Southeast Asia, Europe, and North America [3].

Dengue virus (DENV) is a positive, single-stranded RNA virus from the Flavivirus family, as also seen with Zika virus (ZIKV). Dengue is classified, out of the three mentioned vector-borne disease in this publication, as the most devastating of all arthropod-transmitted viruses due to the virus having the capacity to infect more than 50 million humans a year in a pool of population expanding over three billion, living in endemic regions where the virus thrives [5].

With the spread of the three viruses, Dengue in 2009 in Florida, to the 2013 spread of Chikungunya through the Caribbean, and now, the ZIKV is making its mark on the tropics with major concerns of it spreading to the Western Hemisphere, including the USA [6•]. Like DENV, Zika is a mosquito-borne, positive-stranded RNA Flavivirus. Although the ZIKV is not as prominent as the other viruses, Zika made its mark in 2015 in Brazil, as the outbreak surprised the international health community [7]. By 2016, Zika had already reached North America and was on the radar of the World Health Organization (WHO), which announced the virus as a "global emergency" with the sudden increase of more serious complications of the virus [7]. Those infected with the virus often present with either no symptoms or self-limiting symptoms, although the virus has seen an increase in a number of individuals that are experiencing more severe symptoms, including microcephaly and Guillain-Barré syndrome (GBS) [2]. The transmission of the virus is mainly through the Aedes mosquito; however, it can also be transmitted to the fetus of an infected pregnant woman, as well as through sexual transmission from one infected individual to their partner, even after the initially infected person has recovered from the virus. This puts mothers who travel to the tropics during pregnancy at a higher risk of contracting the virus, which could lead to solemn outcomes to their newborns, including fetal death, placental insufficiency, fetal growth restriction, and microcephaly [7]. With no current vaccines available, travelers, specifically expectant mothers, need to be educated on the risk factors of traveling to certain areas where this virus is prevalent.

Researchers are now considering outside sources like global temperatures as another cause of the spread of the Aedes mosquitoes, as well as ineffective vector control measures. With lack of vaccines, it makes the risk of future spread of these viruses more dangerous, as it will not only affect the tropics but also develop geographical areas [5]. Collectively, there are no current vaccines or antiviral drugs against these three viruses, and with no sign of the spread slowing down, more geographic regions are in danger of being hit by these diseases in the near future. The race to a vaccine has guided this research to further compare and contrast Chikungunya, Dengue, and Zika vector-borne viruses for their similarities and differences.

\section{Methodology}

An electronic literature review search was performed on PubMed, Google Scholar, and MedLine Plus. The search was limited to peer-reviewed articles published from January 1, 2009, until 2018. The articles were selected if the publication included keywords, such as CHIKV, DENV, ZIKV, vector-borne illnesses, mosquito vectors, and vaccinations and treatments associated with Chikungunya, Dengue, or Zika. Articles were then reviewed and included based on the applicability to the topic.

\section{Chikungunya Virus}

\section{Epidemiology of Chikungunya Virus}

CHIKV is an arthropod-borne Alphavirus belonging to the Togaviridae family. It is an envelope, single-stranded positive-sense RNA virus. CHIKV is transmitted from an infected Aedes species mosquito causing Chikungunya fever (CHIKF). The first identified outbreak of human infection with CHIKF was reported back in July 1952 from the southern province of current Tanzania [8]. The clinical signs and symptoms of CHIKF usually begin 3 to 7 days after a mosquito bite and include non-specific flu-like symptoms, a distinctive rash accompanied by lasting joint pain that may persist even after resolution from the infection [9].

Epidemics of symptoms related to CHIKF in humans can be dated back to the 1820s from India, Africa, Caribbean, and West Indies [8]. However, no confirmation could be made as CHIKV infection has many clinical signs and symptoms similar to those of Dengue fever (DF) and Ross River Infection [10]. The epidemic outbreaks were interspersed with periods of disappearance that can range from several years to even a few decades. During epidemics, humans serve as the reservoirs for CHIKV, and during inter-epidemic periods, several vertebrates, such as monkeys, rodents, birds, and Aedes spp. mosquitoes, are the reservoir in parts of Asia and Africa. The first documented Arbo-borne CHIKV outbreak was reported from modern-day Tanzania and has since been the etiologic agent of sporadic epidemics in Africa and Asia, and after 2004, it extended to the islands around the Indian Ocean, Italy, France, and the Americas [8].

Togaviridae family consists of two genera, including Alphavirus and Rubivirus. The alphaviruses are further 
categorized into either New World or Old World groups based on their serologically differentiated complexes [11]. The Old World alphaviruses include Sindbis virus, CHIKV, O'nyongnyong virus, Mayaro virus, Ross River virus, Barmah Forest virus, and Semliki Forest virus. Alphaviruses contain a single strand of positive-sense RNA genome assembled within an icosahedral capsid. The positive polarity RNA genome encodes for four non-structural proteins (NSP1-4) and three structural proteins composed of a capsid protein and two envelope glycoproteins (C, E1, E2) [10]. The virus is associated with a phospholipid envelope that is derived from the host cell membrane during the process of viral maturation [10].

RNA viruses are prone to genetic variations due to viral RNA-dependent RNA polymerase failure to proofread and correct error during replication. The frequent incorporation of wrong nucleotides gives rise to heterogeneous populations of mutant viral RNAs, which is typical of alphaviruses, including CHIKV [10]. The genetic diversity leads to geographically unique lineages that are capable of changing the vector or host specificity. Phylogenetic analysis has revealed three distinct groups based on partial sequences of NS4 and E1 genes: (1) the West African, (2) the East-Central-South African, and (3) the Asian [8]. Populations may contain a variant due to competitive advantages, such as the ability of the virus to replicate in higher titers in a mosquito or vertebrate host or its ability to extend its host range due to mutations [8].

CHIKV is transmitted through two different cycles, including an urban and a sylvatic cycle. Transmission from infected human to mosquito to human is referred to as the urban cycle, while the sylvatic transmission is from animal to mosquito to human. The sylvatic cycle is the primary form of maintenance of CHIKV in Africa. In more densely populated areas, humans act as major hosts, while the genus Aedes mosquitos act as the vector, maintaining an urban cycle of CHIKV [12]. Ae. aegypti is considered as the principal vector for CHIKV among humans; however, Aedes albopictus (Asian tiger mosquito) has also emerged as an important vector [13]. Ae. aegypti is confined within the tropics and sub-tropics, while Ae. albopictus occurs in both warm and cold temperate regions. Strains of CHIKV isolated from A. albopictus during the Indian Ocean's 2005 outbreak have been identified to acquire a mutation in E1 glycoprotein. An alanine to valine mutation at position 226 in the E1 envelope glycoprotein (E1-A226V) increased the fitness of CHIKV [8]. Ae. albopictus mosquito has a widespread distribution and is abundant in Europe and the USA. The mutation has led to improvement in viral survival in the Ae. albopictus population, which in turn leads to the expansion of CHIKV to places where it was once rare, including the Americas and Europe [10].

Since 2005, CHIKV has emerged in Africa, Indian Ocean islands, Asia, Europe, and the Americas [14]. From 2006 through 2010, CHIKV was only confirmed in the USA among people who traveled from affected areas, including Africa and Asia/Southeast Asia [10]. However, in October 2013, two laboratory-confirmed, autochthonous CHIKV cases were detected in the French Territory of Saint Martin Island [8]. According to Centers for Disease Control and Prevention (CDC) (2015), a total of 2811 CHIKV cases were reported in to the USA in 2014. Of those, 12 were locally transmitted cases reported from Florida [15]. All other cases occurred in travelers returning from affected areas. Most CHIKV outbreaks occur during the tropical rainy season where climates allow for mosquito breeding and abate during the dry season. CHIKV is capable of large outbreaks with high attack rates and can affect one-third to three-quarters of the populations in areas where the virus is circulating [12]. Since the first reported CHIKV case in Saint Martin, the virus has spread to over 45 territories in North, Central and South America causing more than 2.9 million suspected local cases and 296 deaths as of late July 2016 [8].

\section{Transmission of Chikungunya Virus}

Mosquitoes become infected when they feed on a person already infected with the virus. After the viral incubation period in the mosquito, infected female mosquitoes then spread the virus to other people during probing and blood feeding. These mosquitoes can be found biting both during the day or night hours, and both species have been actively found biting outdoors and indoors [8]. Following intradermal inoculation by infected mosquitoes, CHIKV directly enters the subcutaneous capillaries where its replication starts immediately. Once in the blood, the virus has access to various parts of the body, including the liver, muscle, joints, and the brain, which eventually leads to symptoms of CHIKF [12].

Vertical maternal-fetal transmission has been documented in pregnant women affected by CHIKF [9]. It has been reported that in the first trimester of pregnancy, direct impact of the infection is associated with a higher risk of spontaneous abortion [8]. There is also the risk of intrapartum transmission, and cesarean section does not appear to prevent the transmission of CHIKV. Signs and symptoms associated with neonatal CHIKF are fever, poor feeding, pain, distal edema, various skin manifestations, seizures, meningoencephalitis, and echocardiograph abnormalities [8].

\section{Clinical Manifestations of Chikungunya Virus Infection}

CHIKF affects all age groups and both genders equally. The disease, however, has a more severe effect on neonates and the elderly [12]. The incubation period ranges from 3 to 12 days before symptoms appear. The course of the disease can be divided into acute and chronic stages. In the acute stage, the onset is usually abrupt and sudden with high-grade fever 
(usually $102-105^{\circ} \mathrm{F}$ ), severe arthralgias, myalgias, and skin rashes. The acute symptoms of CHIKF typically resolve within 7 to 10 days. Chronic stage, also known as the persistent stage, can last for months to years after the initial infection and can present with bilateral, symmetric, debilitating joint pain. Occasional ophthalmic, neurological, and cardiac symptoms can also accompany the chronic stage of infection [12].

The polyarthropathy, including polyarthralgia, polyarthritis, or tenosynovitis of CHIKF, frequently involves the small joints of the hand, wrist, and ankles and the larger joints, such as the knee and shoulder. Joint pains usually resolve in 1 to 3 weeks; however, arthritis can persist in many patients characterized by unpredictable relapses for months to years. Chikungunya is rarely fatal; however, cases of death have been reported. Currently, no specific antiviral treatment, vaccine, and preventive drug exist for CHIKV [11].

Treatment is palliative and includes rest, fluids, analgesics, and antipyretics. Non-steroidal anti-inflammatory drugs (NSAIDs), glucocorticoids, or disease-modifying anti-rheumatic drugs (DMARD) therapy can be used to manage longterm joint pain. Preventative strategies include avoiding outdoors in endemic locations, use of air conditioning, having intact screens on windows and doors to prevent mosquitoes from coming indoors, using insect repellents, and wearing long sleeves shirts and pants when outdoors [16]. Patients suspected of CHIKV should further be protected from another mosquito bite during the first week of illness to prevent further viral spread to other humans [16].

\section{Diagnosis of Chikungunya Virus}

The Aedes mosquito can transmit several other arboviruses. Outbreaks of DF and CHIKF are known to occur frequently presenting with similar symptoms. The differential diagnosis of CHIKV infection varies depending on the place of residence, travel history, and exposures. It is impossible to distinguish these conditions from one another based only on clinical symptoms; therefore, laboratory testing is essential to distinguish CHIKF from other conditions. During the first week of symptoms, reverse-transcriptase polymerase chain reaction (RT-PCR) can be used to detect serum CHIKV RNA with variable sensitivity. An enzyme-linked immunosorbent assay (ELISA) can also be done on patient serum 5 days or more after symptoms begin to evaluate for virus-specific IgM antibodies.

\section{Future Vaccine for Chikungunya Virus}

Exportation of CHIKV from infected travelers is known to have caused Italy's 2007 outbreak [17]. The outbreak of CHIKV in a non-tropical region, including other parts of Europe, has elicited concern and worry for future global threats from the virus. CHIKV transmission relies on urban cycle and leads to high chances of outbreaks and long-term debilitating arthralgia. Control of transmission requires control of the mosquito vector and prevention of bites from infected individuals, which can be very difficult to achieve. Reemerging outbreaks of CHIKV has sparked many kinds of research into prevention methods. There are currently many CHIKV vaccine candidates in pre-clinical and clinical development stages. Only two candidates thus far have qualified to enter phase II of clinical trials: a CHIKV-like particle-based vaccine and a recombinant live attenuated measles virusvectored vaccine [17]. Humoral memory host response and the presence of neutralizing antibodies targeting the virions' outer surface glycoproteins are the primary form of protection generated against CHIKV [17]. Therefore, the goal is to ensure that a vaccine is able to elicit a strong immune response to protect from future outbreaks.

Protein subunits and inactivated vaccines are traditionally considered the safest form of immunity. One method being tested is a combination of adjuvant CHIKV envelope proteins. This approach has shown to generate a short-lived immunity requiring multiple doses and providing only partial protection from the virus [8]. Also, manufacturing a high biocontainment and ensuring the total inactivation of the virus proved to be costly and a major concern with vaccine development. Virus-like particles (VLP) tend to be more immunogenic and are equally safe. An experimental vaccine, developed by investigators at the National Institute of Allergy and Infectious Diseases (NIAID's) Vaccine Research Center, is currently being tested in a phase II trial [18].

Live-attenuated vaccines against CHIKV have progressed the furthest into clinical trials. The vaccine was created from a CHIKV isolate to generate an attenuated virus strain. In humans, this vaccine proves to be highly immunogenic, but some strains also have caused arthralgia in certain individuals, leading to a concern about incomplete viral inactivation.

Another strategy in CHIKV vaccine development is the use of vaccine vectors, like the measles virus. In this approach, the structural CHIKV genes are inserted into the vector's genome, which initiates the expression of CHIKV structural proteins upon infection. Preclinical studies of the live attenuated measles virus-vaccine expressing CHIKV envelope and capsid proteins have been shown to induce a robust neutralizing immune response, which completely protects the experimental mice [8]. In the clinical trial phase, the vaccine has shown to increase antibodies providing long-term protection with no serious adverse effects. Several CHIKV vaccine candidates appear to provide promising protection against CHIKV. A single vaccine could possibly provide worldwide protection; however, no vaccine against CHIKV disease has yet undergone efficacy testing in humans. Vaccine-efficacy against CHIKV infection can only be determined in large scaled clinical trials, including people at risk of CHIKV infection in affected countries. 


\section{Dengue}

\section{Epidemiology of Dengue Virus}

DF is one of the world's most important mosquito-borne viral diseases [19]. A recent estimate reports that approximately 390 million DENV infections occur every year [20]. Of those infections, 96 million manifest clinically with varying degrees of severity [20]. Additionally, Dengue has been estimated to cause an annual burden of 750,000 disability-adjusted life years and approximately 24,000 deaths, of which the majority occur in children [19]. An accurate number of cases have not been identified because of misdiagnosis, underreporting, and over reporting of cases [21]. In the last 30 years alone, it has been reported that the number of countries with DF outbreaks has increased tenfold [22]. The viral illness has re-emerged in countries that had once eradicated the disease [19]. Despite this knowledge, DF has been ranked fifth on the list of neglected tropical diseases in the Americas [23].

\section{The Vectors and History of Dengue Virus}

Today, DF cases are reported across countries in the AsianPacific, the Americas, the Middle East, and Africa and continue to rise globally [24]. However, the vectors responsible for the dissemination of the virus, the Ae. aegypti and Ae. albopictus mosquitoes, were once restricted to regions of Africa and Asia. The Ae. aegypti, once referred to as the African mosquito, is an urban-dwelling anthropophilic mosquito [5]. In contrast, the Ae. albopictus mosquito, also known as the Asian tiger mosquito, is rural-dwelling [5]. The worldwide spread of DENV was led by the global spread of the Ae. aegypti and Ae. albopictus mosquitoes [25]. It has been postulated that the onset of the slave trade in conjunction with harsh environmental conditions allowed for the introduction of the Ae. aegypti into the Americas, from where the Ae. aegypti spread to the tropical and subtropical regions of the world [25]. In areas where the Ae. aegypti is not endemic, the Ae. albopictus, whose origins were as forest species from Asia, is the primary vector of DENV. The Ae. albopictus spread to islands in the Indian and Pacific Oceans during World War II due to movements of army soldiers [23]. More recently, the Ae. albopictus has been connected to outbreaks of DF in Hawaii, Central Africa, and China [23].

Historically, in the Americas, Benjamin Rush was the first to clinically describe DF in the 1770s in Philadelphia [26•]. From the 1950 s to the 1970 s, the Americas had eradicated the Ae. aegypti with a continent-wide vector control campaign [19]. This vector eradication led to virtually no cases of DF. However, by the 1970 s, the continent-wide vector control campaign had come to an end, and a sudden rise in urbanization led to the return of the Ae. aegypti mosquitoes and with them DF [19]. In the past couple of decades, tropical areas of
Central America, South America, and the Caribbean have experienced a substantial increase in reported cases of DF [19]. In fact, in a 5-year span in the early 2000 s, there were almost one million more cases officially reported of DF in the Americas than there were for the period of 1995 to 1999 [19].

In Europe, in the nineteenth and twentieth centuries, cases of DF were reported throughout the Mediterranean [27]. In particular, DF outbreaks were reported in Athens, Greece multiple times between 1889 and 1910 [27]. By 1927, Athens and its surrounding areas were the origins of the last major severe DF outbreak on the continent of Europe [27]. The epidemic lasted until 1928 with a reported number of approximately 650,000 cases [27]. The virus made an unexpected reappearance in Europe in 2010, with two cases of DF identified in Nice, France [27].

\section{Factors Influencing the Spread of Dengue Virus}

There are numerous dynamics that influence the spread of DF virus. These factors include, but are not limited to, the vector distribution, human travel, urbanization, and climatic changes [28]. The increase in human population density, the mobility of populations, urbanization, modern transportation, and an increase in commerce steered the reemergence of DF [28]. An increase in vegetation, tree cover, housing quality, and surrounding land area is also associated with increased cases of Dengue [5]. Climate is another contributing factor for the spread of viral diseases. Climate not only affects the interactions between humans and the vector but also has been shown to influence vector dynamics, such as vector development rates, mortality, behavior, and viral replication within the vector [29]. Additionally, the Ae. aegypti and Ae. albopictus mosquitoes have adapted and evolved to living near areas of human habitation by becoming independent of the need for reservoir environments serotype [5]. Finally, viral strain virulence and genetic variation increase the risk of infection and severe illness.

\section{Nomenclature and Viral Genetic Diversity of Dengue Virus}

Dengue is derived from the Caribbean word meaning "affected" and is first used to describe outbreaks of the illness in the Caribbean in 1635 [23]. The DENV belongs to the genus Flavivirus of the family Flaviviridae [23]. It is an icosahedral, enveloped, single-stranded, positive-sense RNA virus whose core is 40 to $50 \mathrm{~nm}$ in diameter and contains the $\mathrm{C}$ protein that encapsulates the viral genome [29]. It is composed of the capsid, membrane, and envelope, which are each a structural protein. The viral lipid envelope, which surrounds the core, itself, is composed of two proteins, $M$ and $E$ [29]. It is also comprised of seven non-structural proteins (NS), which are NS1, NS2a, NS2b, NS3, NS4a, NS4b, and NS5 [30]. The 
viral illness is due to infection by one of the four viral serotypes that share antigenic relationships: DENV-1, DENV-2, DENV-3, and DENV-4 [31]. The most frequently isolated serotypes in reported DF cases of the twentieth century were DENV-1 and DENV-2, and in the twenty-first century, are DENV-2 and DENV-3 [24]. Infection with a particular serotype affords lifelong protection against reinfection with that serotype; however, it does not afford protection against a secondary infection with a different serotype [5]. Secondary infection with a different serotype may in fact enhance the seriousness of the disease [5].

\section{Mode of Transmission for Dengue Virus}

As the mosquito feeds on the human host, it injects the DENV into the bloodstream and surrounding epidermis and dermis, which results in the infection of immature Langerhans cells and keratinocytes through receptor-mediated endocytosis [29]. The Ae. aegypti feeds on human hosts during daylight hours and rests indoors, whereas the Ae. albopictus is exophagic and feeds on human hosts opportunistically [25]. An extrinsic incubation period (EIP) describes the number of days required between the time a vector acquires an infectious pathogen and when it is capable of being transmitted to a subsequent host [32]. The EIP is an important component of the transmission of the DENV [5, 32]. Pathogen transmission of the DENV can only occur if the vector(s), Ae. aegypti and Ae. albopictus mosquitoes bite a host after sufficient time for replication of the virus, which is generally considered to be eight to 12 days $[5,32]$.

\section{Clinical Manifestations of Dengue Virus Infection}

Infection with any DENV serotype can lead to various forms of illness ranging from unapparent and asymptomatic to severe and fatal. Infections may present as asymptomatic, undifferentiated acute febrile illness (AFI), DF, or severe DF, which most often occurs among those with secondary DENV infections or among children under the age of one year of age, whose mothers were previously infected with DENV [33]. Severe DENV infections can be categorized as either Dengue hemorrhagic fever (DHF) or Dengue shock syndrome (DSS) [33]. Approximately half of the individuals infected with DENV are asymptomatic, and additional large percentages have non-specific, benign symptoms that are selflimiting [26•]. The asymptomatic and mild presentations are often associated with primary infections. DF is predominately self-limited to 1 week with a presentation that includes 2 to 7 days of high fever and two or more of the following symptoms: a frontal headache, myalgias, arthralgias, and a diffuse maculopapular cutaneous erythematous rash $\left[21,26^{\bullet}\right]$. Other manifestations of the illness may include mild hemorrhagic symptoms, such as easy bruising, petechiae, epistaxis, gingival bleeding, and leukopenia [34]. The classic presentation of DF is often seen in children over the age of 15 and in adults. DHF and DSS infections present with hypotension, plasma leakage, hemorrhage, thrombocytopenia, and alterations in neurologic function [21]. DHF is classified into four grades: I and II, which represent relatively mild cases without shock, and III and IV, which are more severe, accompanied by shock and considered to be DSS [34]. DSS occurs when profound shock sets in with a rapid, weak or thready pulse, hypotension, and cold, clammy skin (grade III) or with an undetectable pulse (grade IV) and is frequently fatal due to the extensive increase in capillary permeability and plasma leakage [24].

\section{Diagnosis of Dengue Virus}

The critical diagnosis of the array of diseases associated with DENV can be made early and accurately through various clinical and laboratory methods. The clinical symptoms associated with DF vary widely; hence, laboratory diagnosis is needed for confirmation. The WHO [35] proposed that an infection be classified as probable AFI or DF when it includes two or more of the following: headache, retro-orbital pain, myalgia, arthralgia, rash, hemorrhage, and leukopenia; and supportive serology of a reciprocal hemagglutinationinhibition antibody titer of greater or equal to 1280 , which is comparable to an IgG ELISA titer, or a positive IgM antibody test on a late acute or convalescent-phase serum specimen. The infection can be classified as a confirmed case when there are corroborating laboratory criteria for confirmation of DF, including the isolation of the virus, the viral nucleic acid, antibodies, and antigens, or through a combination of the aforementioned techniques. Specifically, the laboratory criteria must include one of the following: isolation of the DENV from serum or autopsy; a fourfold or greater change in reciprocal IgG or IgM antibody titers to DENV antigens in paired serum samples; DENV antigen present in autopsy tissue, serum, or cerebrospinal fluid samples via immunohistochemistry, immunofluorescence, or ELISA; or the detection of DENV via polymerase chain reaction (PCR) [35]. In order for DHF/DSS to be diagnosed, all of the following criteria must be met: (1) fever, which may be biphasic, lasting 2 to 7 days; (2) hemorrhagic symptoms confirmed by a positive tourniquet test, petechiae, bleeding from the mucosa, hematemesis, or melena; (3) thrombocytopenia of 100,000 cells per $\mathrm{mm}^{3}$ or less; and (4) evidence of plasma leakage manifested by a rise in hematocrit greater than or equal to $20 \%$ above the average for sex and age, a drop in hematocrit following volume replacement treatment greater than or equal to $20 \%$ of baseline, or other signs of plasma leakage such as pleural effusion, ascites, and hypoproteinemia [35].

The differential diagnosis includes a wide-ranging list of various infections [35]. Of these possible differential 
diagnoses, the most difficult to differentiate clinically from $\mathrm{DF}$ is CHIKF [35]. Clinically, shock rules out a diagnosis of CHIKF. Laboratory findings help establish a diagnosis early and accurately. The laboratory findings of thrombocytopenia help distinguish DHF/DSS from other illnesses that can present with shock, including bacterial infections that exhibit endotoxin shock or meningococcemia [35].

\section{Treatment and Prevention of Dengue Virus}

Treatment for DENV infections is in large part only supportive. Treatment includes symptomatic control with close monitoring of hemodynamics, any possible hemorrhages, and volume status. As previously mentioned, early and accurate confirmation of a Dengue illness is vital in order to avoid the possible complications of shock [26•]. The mortality rate for DENV infection is reported to decrease dramatically with good supportive care [26•]. The strategies for preventing mosquito bites normally focus on diminishing the concentration of the vector populations [32]. One of the main ways to control the vector population is to reduce the availability of larval habitats by diminishing areas of stagnant water stores and diminishing areas of collected solid waste [32]. In addition to large-scale efforts to eliminate the mosquito vector responsible for the spread of DENV, individual precautions may be taken in areas where DENV infections are endemic or where recent outbreaks have been reported. In order to protect against mosquito bites, individuals should wear protective clothing, including long pants and long sleeve shirts, insect repellent, and use of other protective garments, such as mosquito netting [26•]. The most effective form of prevention is by preventing mosquito bites, due to the fact that currently, there is no vaccine or specific treatment options available for DENV infection [26•].

\section{The Race to Dengue Vaccine}

The continuously rising incidence rate of Dengue in the recent decades has fueled advancements towards a Dengue vaccine. The goal of vaccination is to decrease the transmission of natural DENV to an extent where DENV infections are almost nonexistent. However, there are some critical issues in Dengue vaccine development, due to the DENV serotypes and the specific function of memory $\mathrm{T}$ cells in the immune response in those infected with DENV [36]. Serotype diversity has been associated with limiting the efficacy of monoclonal antibody therapy and of tetravalent vaccines against DENV [5]. Despite these challenges, the search for an efficacious and possibly safe vaccine continues. Current research and trials of Dengue vaccines include live attenuated vaccines, live attenuated vaccines using infectious clone technology, genetic vaccines using virus and plasmid vectors, and many recombinant subunit vaccines [5].
The Sanofi Pasteur live attenuated vaccine, which is a chimeric Yellow Fever Virus (YFV)-DENV tetravalent Dengue vaccine (CYD-TDV), is currently the most advanced vaccine with known efficacy and results [37]. In the CYD-TDV vaccine, the backbone of the YFV strain 17D attenuated vaccine was used, and the structural genes encoding for the $\mathrm{M}$ and $\mathrm{E}$ proteins of YFV were replaced with the $\mathrm{M}$ and $\mathrm{E}$ proteins for all four DENV serotypes [37]. Thus, the replication machinery of the virus is that of YFV, and the outer structure is of a DENV serotype [37]. This leads to CD4+ T cell response against DENV and a CD $8+\mathrm{T}$ cell response against YFV [37]. The phase I and II trials illustrated that the vaccine was safe and tolerable in humans [37]. Phase III trials reported an efficacy range of 51.1 to $79 \%$ in Southeast Asia and 31.3 to $77.5 \%$ in South America [37].

Many other vaccines are also currently in the early developmental stages. TetraVax is another live attenuated DENV vaccine that employs the deletion of thirty nucleotides from the 3' UTR of DENV. The formulation includes four admixtures (TV001 to TV004) of rDEN1 $\Delta 30$, rDEN2/ $4 \Delta 30(\mathrm{ME}), \mathrm{rDEN} 3-3{ }^{\prime} \mathrm{D} 4 \Delta 30$ or $\operatorname{rDEN} 3 \Delta 30 / 31-7164$, and rDEN4 $\Delta 30$ or rDEN4 $\Delta 30-200,201$ [38]. Depending on the formulation, the results of phase I clinical trial illustrated tri- or tetravalent adequate antibody response after a single dose in 75 to $90 \%$ of participants [38]. Admixture TV003 (rDEN3 $\Delta 30 / 31, \operatorname{rDEN} 4 \Delta 30, \operatorname{rDEN} 1 \Delta 30$, and rDEN2 $/ 4 \Delta 30$ ) has been proposed for a phase II trial, because it produced a trivalent antibody response in $90 \%$ of the participants, of which $45 \%$ had a complete tetravalent antibody response [38].

In addition, there were two recent phase I trials for purified inactivated DENV1 vaccine, DENV-1 PIV, and a tetravalent purified inactivated DENV vaccine, TDENV PIV [38]. The monovalent DENV-1 PIV was deemed safe in the phase I study [23]. TDENV PIV has been shown to induce a longlasting antibody response in nonhuman models [23].

Many recombinant subunit vaccines are also in various stages of pre-clinical and early clinical development. These recombinant subunit vaccines are primarily based on the DENV E protein (80E). Multiple expression systems for the production of recombinant $\mathrm{E}$ protein have been explored, including but not limited to Saccharomyces cerevisiae, E. coli, Pichia pastoris, and stably transformed Drosophila S2 cells, which are capable of expressing $80 \mathrm{E}$ in high levels in its native form [38]. DENVax, which is a recombinant live attenuated tetravalent vaccine, was deemed safe during phase I testing [23]. DENVax substitutes the $M$ and $E$ proteins of the DENV2 PDK-53 vaccine, which was attenuated by serial passage in non-human kidney cells and has been studied in clinical trials as a monovalent vaccine candidate as well as in multivalent formulations, with the $\mathrm{M}$ and $\mathrm{E}$ proteins of wild-type DENV$1,-3$, or -4 [38]. The vaccine is currently undergoing a phase II trial in children and adults [38]. Another tetravalent subunit 
vaccine currently in phase I testing is V180 also includes the 80E subunit [38].

In spite of all the current promising clinical trials for DENV vaccine, a fully effective vaccine is yet to be developed. Further research is needed to establish a better understanding of the basis for Dengue pathogenesis and the immune response to DENV, not only to improve our understanding of the immune response to the different serotypes of DENV, especially in association with secondary infections, but also to enable the ability to create a safe and fully effective vaccine.

\section{ZIKA}

\section{Epidemiology of Zika Virus}

Although ZIKV now circulates throughout most of the Americas, South East Asia, and the Pacific Islands, ZIKV was first discovered in Uganda in 1974 and is a member of the genus Flavivirus in the family Flaviviridae [39]. The virus was initially restricted to the equatorial zones of Africa and Asia until 2007 when it first emerged in Yap, an island located in the Caroline Islands of the western Pacific Ocean [2]. This made it even easier for further spread eastward towards French Polynesia, other Pacific Islands, and Latin America from 2013 to 2015, eventually spreading to North America in 2016 [2]. The first isolation of the ZIKV was from the serum of a rhesus macaque monkey in 1947 [2]. The monkey resided in the Zika forest on the Entebbe peninsula in Uganda [2, 39, 40]. The strain (MR-766) obtained from this monkey was the African prototype strain of ZIKV. It was after this that the ZIKV was recovered from mosquitoes caught in the same area on the Entebbe peninsula [2, 41].

At the time, there was no evidence that this virus caused any form of illness to the human population around the peninsula, and the antibody to ZIKV was found in about 10 to $20 \%$ of the local population [2]. Since the discovery of the virus, several other studies have shown that the burden of illness caused by the virus on human population is distributed relatively narrowly along the equatorial belt from Africa to Asia [42]. The first incidence of human disease caused by ZIKV was first reported in Nigeria in 1954 [41]. The infection was confirmed in three people by the isolation of the virus and by an increase in the level of serum antibody against the ZIKV [41]. Since 1954, there has only been about a dozen Zikaassociated illness incidence in Africa and Asia [41]. It wasn't until 1966 when the first non-African strain (P6-740) was isolated from Ae. aegypti mosquitoes in Malaysia [2]. The first large outbreak outside of Africa and Asia was on Yap Island with a disease characterized by fever, rash, arthralgia, and conjunctivitis [43]. During this outbreak, as many as $73 \%$ of the residents of Yap were infected and as many as $18 \%$ showed clinical symptoms that were most likely related to infection by ZIKV [43]. Furthermore, it was later discovered that the ZIKV was introduced to the island from Southeast Asia. In early to late 2010, a number of sporadic cases of ZIKV were reported in countries in Southeast Asia, such as Thailand, Cambodia, Malaysia, Indonesia, and the Philippines [7].

The French Polynesia outbreak from 2013 to 2014 was a major epidemic [44] with approximately $11 \%$ of the total population presenting with symptoms and sought treatment for the suspected ZIKV infection [2]. Although the origin of the ZIKV during the French Polynesian outbreak is still unknown, the majority of the clinical manifestations were similar to those seen in the 2007 Yap outbreak [2, 45]. Furthermore, the strain of virus in the French Polynesian outbreak is genetically similar to the strain recovered on Yap in 2007. There was an array of neurological manifestations during the French Polynesian outbreak, which included GBS [46]. After the French Polynesian outbreak, Zika spread to other neighboring islands in the South Pacific Ocean and also other countries even further away such as Australia, Italy, Japan, and Norway [46].

At the beginning of 2015, ZIKV was detected in the northeastern part of Brazil [47], and by the end of 2015, it was detected in at least 14 states throughout Brazil with cases estimated to be between 440,000 to $1,300,000$ [2]. It was noted that the number of cases of microcephaly had increased in suspected Zika-infected regions, and by February of 2016, there were over 4000 suspected cases of microcephaly [2, 47]. In 2015, Colombia reported a local outbreak of ZIKV infection, and by March 2016, there were a total of 51,473 suspected with ZIKV infections reported in Colombia alone [47]. ZIKV has spread throughout Central and South America, as well as to the Caribbean islands. There is alarming evidence of a correlation between ZIKV infection and microcephaly, a condition where the head circumference is smaller than normal, which has prompted the WHO to declare a "public health emergency of international concern" [46, 48]. ZIKV is still causing ongoing concerns in parts of Latin America, North America, and the rest of the world. As of November 2016, there has been a plethora of incidences of mosquito-borne transmission of the ZIKV reported throughout the Americas and other locations [2].

\section{Genetic Diversity of Zika Virus}

Since ZIKV was first discovered in 1947, there has been approximately over $500 \mathrm{ZIKV}$ isolates that have been identified throughout Africa, Asia, the Pacific Islands, and the Americas [39]. Despite the number of isolates that have been identified, only a handful of strains have been sequenced. The phylogenetic analysis of all the 29 completed or nearly completed strains show that the distinct ZIKV strains can be divided into two geographical areas based on genetic lineages: African and 
Asian lineages $[2,39,40]$. Furthermore, the spread and transmission of the virus can be linked to an increase in air travel from Zika-endemic area to areas like Brazil and the Pacific Islands [43]. More research is needed to broaden our understanding of the evolution, diversity, and burden of illness caused by different strains of ZIKV.

\section{Transmission of Zika Virus}

\section{Vector}

Since the 1947 discovery of ZIKV in sentinel monkeys in Uganda and the isolation of the virus from the Aedes africanus mosquitos 1 year later, there has been several reports of Aedes species harboring the ZIKV [40]. For instance, the outbreak on Yap Island is attributed to the Aedes hensillii, while the outbreak in French Polynesia was attributed to Aedes polynesium. Furthermore, the outbreak in Brazil is attributed to the Ae. aegypti and Ae. albopictus [40, 42]. Interestingly, the Ae. aegypti was identified as the cause of the Malaysia 1969 outbreak. Additionally, it was also implicated in French Polynesia and Indonesia [40]. Interestingly, it has been observed that the Ae. aegypti, which is most prevalent in areas of Africa, is being replaced by its Asian lineage counterpart; Ae. albopictus, the Asian counterpart, was the predominant vector implicated in the Gabon outbreak during 2007 [42].

The ability of these mosquito species to adapt to harsh and different environments, breed ubiquitously, and survive various climates and their ability to disperse passively by humans, in addition to the extensive trends of human urbanization, traveling, and changing of weather patterns within the last decade, make it impossible to rule out the risk of impending epidemic outbreaks [42]. It will be inadequate to assume the implicated vector in the case of an outbreak. The vector competence needs to be assessed against the vector capacity, which is the concept that explains the chance of effective human-vector contact and transmission [40]. The viral capacity does not depend only on the innate vector characteristic, but other factors like host range, the local density of the vector, feeding behavior, biting and survival rate, and colonizing success $[40,49]$. This being said even the presence of a particular vector species does not confirm that the species is the primary vector $[2,40]$.

\section{Non-vector}

In 2011, the first case of sexual transmission of ZIKV was reported [50]. Since then, several studies have shown evidence of unprotected oral, anal, and vaginal intercourse leading to sexual transmission of ZIKV [40]. More researches have been published that supports the sexual transmission of ZIKV. In 2015, others have reported and identified that the
ZIKV RNA as well as the infectious virus strains were isolated from semen $[51 \cdot, 49]$.

Previous studies report that ZIKV RNA had been isolated from breast milk of three symptomatic mothers, although more recent reports identified ZIKV particles found in the breast milk of one asymptomatic mother [49]. Evidence of non-sexual contacts, as described by researchers [40, 49] in 2016, suggests one of the ways the burden of the illness caused by ZIKV is transmitted. Blood transfusions have also been implicated as a mode of transmitting Zika viral particles.

\section{Clinical Manifestations of Zika Viral Infection}

ZIKV has been known to cause a variety of clinical symptoms in patients; some of which include, joint pain, rash, conjunctivitis, headache, and muscle aches [49]. These symptoms are only present in about 18 to $57 \%$ of cases, and it is relatively uncommon for these symptoms to lead to hospitalization. However, in severe cases, ZIKV has been shown to cause neurological and ophthalmic complications, which include GBS in adults and microcephaly in fetuses and newborns [2, 40, 49]. Other clinical manifestations observed with acute ZIKV infection include hematospermia, a condition which causes blood in semen [51•].

Although there is compelling evidence that Zika viral infection causes severe neurologic complications, these manifestations have only been recently described. The frequency of direct central nervous system (CNS) involvement by ZIKV infection is of research significance. Cases of CNS encephalopathy syndromes and seizures due to ZIKV infection have been reported. In a recently published report [52], an 81-yearold man who had been on a 10-day cruise to the Pacific Islands had returned home only to be diagnosed with meningoencephalitis. The cruise-goer was admitted to the intensive care unit (ICU) due to fever, a decreased level of consciousness, and was found to have a positive RT-PCR for Zika in his cerebrospinal fluid [52]. GBS is a disease of the immune system that is characterized by the production of antibodies against components in the peripheral nervous system (PNS), which results in the weakness and tingling of the body in an ascending fashion [51•].

ZIKV infection causing GBS was first reported in the 2013 to 2014 outbreak that affected the French Polynesian islands [51•]. During this outbreak, the incidence of GBS was estimated to increase by approximately twenty folds per 100,000 per year. Furthermore, recent studies have reported the detection of ZIKV antibodies in patients with GBS. In 2016, a casecontrol study reported the incidence of 42 cases of GBS after a ZIKV infection between October 2013 and April 2014. This data finding is significantly less than the data (five to ten cases) presented in the same period the year prior [53]. Although, the mechanism at which ZIKV causes GBS is 
currently unknown, the symptoms of GBS are only transient, and most patients attain full recovery [2].

ZIKV has been implicated in several cases of microcephaly in newborns [40, 49]. Microcephaly is a condition where the brain of a fetus or infant is relatively smaller than normal and can be described in one or two various forms. Congenital microcephaly is present at birth, while acquired microcephaly develops after birth [49]. The mechanism of formation of both types of microcephaly is different. Primary microcephaly most likely results from the loss of neurons during neurogenesis, whereas secondary microcephaly has been attributed to the dendritic process and synaptic connection after birth [2]. In 2016, researchers reported a study that included 345 pregnant women, all of whom had symptoms of a rash [54]. More specifically, 53\% tested positive for ZIKV in their blood and urine. Of the remaining 126 patients, there were nine fetal deaths and 117 live births. Of the live births, $42 \%$ had altered neuroimaging findings with four confirmed cases of microcephaly and 11 cases of congenital ZIKV syndrome. Furthermore, in the same year, other researchers reported the results of a radio imaging study that compared brain images of patients with confirmed Zika infection and 18 patients with suspected ZIKV infection [55]. This study showed that the brain images between these two groups were similar, showing evidence of ventriculomegaly and intracranial calcification of the gray-white junction, thalamus, and basal ganglia [7].

Ocular lesions in three infants of mothers infected by ZIKV in Brazil were reported by one group of researchers [56]. These infants also presented with microcephaly and cerebral calcifications. Upon examination, none of the infected mothers presented with ocular lesions, but the infants had gross pigmentations in the macula and no foveal reflex. Another published report implicated ZIKV as the causal agent in 40 infants with microcephaly who have eye lesions [56]. Furthermore, this same study reports that there was an increased frequency of ocular lesions in infants of mothers who were symptomatic during the first trimester [56].

\section{The Race to Zika Vaccine Development}

The race to develop a potential vaccine for ZIKV has been imminent since the recent worldwide outbreaks. Although scientists worldwide are still investigating ways to curtail and mitigate the burden of the diseases caused by ZIKV, a lot is known about its relatives, such as West Nile [42]. These vaccines have been created using different strategies: inactivated or live-attenuated viruses, recombinant proteins and recombinant subviral particles expressed in different heterologous systems, chimeric backbone viruses, or naked cDNA [57]. After the widespread of Zika in the Americas, pharma giants have been investigating several vaccine designing strategies that can be used to reduce the burden of diseases caused by Zika.
Over 30 vaccine candidates are in an inactive preclinical development, and few have been approved by the Food and Drug Administration (FDA) for phases I and II of the clinical trial, while some pharmaceutical companies have also completed their phase 1 clinical trial [58]. The development of a vaccine candidate needs to take into account the characteristics of Zika infection. For instance, men might be a target for the infection; however, men might not be a prime candidate for a potential ZIKV vaccine. ZIKV usually attacks pregnant women or women of childbearing age more severely, and a positive infection in this population can lead to congenital abnormalities in the newborns. The vaccine development has presented some concern, particularly in the sense that this potential vaccine may interact negatively with other vaccines already being administered for other Flavivirus infections [42].

\section{Treatment and Prevention of Zika Viral Infection}

Till date, there is no specific treatment available for ZIKV infection [42]. Treatment is modeled towards supportive therapy, including but not limited to rest and the use of oral analgesics, as needed for pain [42]. In order to prevent bleeding and Reye's syndrome, aspirin should not be given to children under the age of 12. Aspirin and other NSAIDs should also be avoided in patients with unconfirmed ZIKV infections, as a precaution in the event that the symptoms are actually caused by Dengue or Chikungunya - two infections in which NSAIDs are contraindicated [42]. Attempts to develop a pharmacological treatment are in research and developmental stage for patients who have been confirmed to have ZIKV infection. Investigators at Utah State University have reported that Ranpirnase, a cancer chemotherapeutic agent, was active in blocking ZIKV compared with the control [42].

In response to the growing evidence that ZIKV infection is sexually transmissible [59], the CDC has recommended the use of latex condoms for people who have recently visited areas where ZIKV is endemic [42]. Blood donations are another potential source of ZIKV transmission [2, 42, 54, 58]; therefore, the FDA recommends that individuals defer donating blood if they have been to areas with active ZIKV transmission, have potentially been exposed to the virus, or have had confirmed ZIKV infection. In areas without active ZIKV transmission, the FDA recommends that donors at risk for ZIKV infection defer donation for a period of 4 weeks [40]. Individuals considered to be at risk include those who have had symptoms suggestive of ZIKV infection during the past 4 weeks, those who have had sexual contact with a person who has traveled to or resided in an area with active ZIKV transmission during the prior 3 months, and those who have traveled to areas with active transmission of ZIKV during the past 4 weeks. In areas with active ZIKV transmission, the FDA recommends that whole blood and blood components 
for transfusion be obtained from areas in the USA without active transmission [40]. Blood establishments may continue collecting and preparing platelets and plasma if an FDAapproved pathogen-reduction device is used. The agency's guidelines also recommend that blood establishments update donor-education materials with information about the signs and symptoms of ZIKV infection and ask potentially affected donors to refrain from giving blood [40]. The FDA has also reported that another mode of transmission of Zika infection is by donation of human cells, tissues, and cellular and tissuebased products (HCT/Ps). HCT/Ps includes corneas, bone, skin, heart valves, hematopoietic stem/progenitor cells (HPCs), gestational tissues (such as an amniotic membrane), and reproductive tissues (such as semen and oocytes) [40].

\section{Discussion and Conclusion}

\section{Similarities and Differences Between Chikungunya, Dengue, and Zika}

Arboviruses have numerous similarities, more specifically in their location and presenting symptoms. However, understanding the differences of the arboviruses is important for diagnosis, as it is not uncommon to misdiagnose and underreport cases. The similarities of these diseases can be seen in their viral family, environment, transmission, symptoms, and treatment. Each of the viruses causing these diseases has its own unique characteristics, complications, and symptoms. Africa, the Pacifics, South-East Asia, and the Americas are some of the primary locations, which consist of the tropical and sub-tropical areas in equatorial regions, affected by Chikungunya, Dengue, and Zika viruses [60]. All three viruses are arthropod-borne. Dengue and Zika belong to the Flavivirus genus of Flaviviridae family, while Chikungunya belongs to the genus Alphavirus of the Togaviridae family. They are all transmitted in a human-to-mosquito-to human cycle. Along with this mode of transmission by an infected mosquito, ZIKV can also possibly be transmitted sexually as there are reported cases of sexual transmission between returned travelers and their significant others [61]. Zika can also be transmitted vertically from an infected mother to fetus transplacentally or during delivery [62].

The transmission vector for these arboviruses is also the same. The Aedes genus mosquitoes especially Ae. aegypti serves as the vector. Other Aedes genus members that can transmit the virus are Ae. hensilli, Ae. scutellaris, Ae. polynesiensis, and Ae. albopictus. The viruses circulate in blood making blood and organ donation transmission possible [63]. Characteristically, Chikungunya, Dengue, and Zika viruses show a lot of similarities as they are all shaped spherically with a diameter of 40 to $70 \mathrm{~nm}$ and 11 - to $12-\mathrm{kb}$ genome in size [64]. The receptor binding and antibody neutralization protein of Chikungunya are different from that of Zika and Dengue, in the sense that it comes from a different viral family [65]. Dengue has four different serotypes, while Zika and Chikungunya have one serotype each. In regard to their signs and symptoms, the similarities between the viruses can sometimes lead to misdiagnosis. As Dengue is the longest known, when Chikungunya was first discovered, differentiating it from Dengue was difficult and led to confusion. This confusion is currently manifesting itself once more in the cases with Zika [66].

Furthermore, $80 \%$ of Zika infections are presumed to be asymptomatic [67]. This is also the case with DF. Contrary to Dengue and Zika, Chikungunya cases are 75 to $97 \%$ symptomatic [11]. Retro-orbital pain, rash, fever, joint pain, lymphadenopathy, and muscle pain are the common symptoms of Chikungunya, Dengue, and Zika (Table 1). These symptoms appear between 2 to 14 days. In addition to these symptoms, each virus has a few unique symptoms. The fever induced by Zika is mild, whereas the fever in Chikungunya can go up to $102.02^{\circ} \mathrm{F}\left(38.9^{\circ} \mathrm{C}\right)$, and the fever induced by Dengue is the worst as it can reach $104^{\circ} \mathrm{F}\left(40^{\circ} \mathrm{C}\right)$ [60]. Rash occurrence and presentation in these diseases are also different. Zika has the highest rash occurrence as it appears in 90 to $96 \%$ of cases in a face to limb spreading fashion [63]. There is a measles-like rash in Dengue, often called "islands of white in the sea of red," and occurs in 50 to $80 \%$ of cases. The rash of Chikungunya appears in the extremities and the trunk [11]. The muscle and joint pains in Zika and Dengue are not as severe as those seen in Chikungunya. The joint pain in Chikungunya can be disabling to those infected. Specific unique symptoms of DF include an acute bleeding phase from the mucous membranes of the mouth and nose and can present in a critical phase as hemorrhage [68]. Viremia in Chikungunya infection is high and causes hepatomegaly [11]. Symptoms specifically attributed to Zika infections are conjunctivitis and edema of the extremities [60].

Table 1 Comparative prevalence of various signs and symptoms associated with Chikungunya, Dengue, and Zika [60]

\begin{tabular}{llll}
\hline Disease & Dengue & CHIK & Zika \\
\hline Fever & +++ & +++ & +++ \\
Myalgia/arthralgia & +++ & ++++ & ++ \\
Oedema in extremities & - & - & ++ \\
Maculopapular exanthema & ++ & ++ & +++ \\
Retro-orbital pain & ++ & + & ++ \\
Conjunctivitis & - & + & +++ \\
Lymphadenopathies & ++ & ++ & + \\
Hematomegaly & - & +++ & - \\
Leukopenia/thrombopenia & +++ & +++ & - \\
Bleeding & + & - & - \\
\hline
\end{tabular}

CHIK: Chikungunya 
Additional research is needed to understand whether population immunity in endemic countries will confer protection against the epidemic strains of Chikungunya, Dengue, and Zika currently circulating.

These viruses have numerous commonalities regarding their transmission and characteristics. Proper diagnosis, treatment, and vaccination can be developed with an understanding of not only their similarities but also, more importantly, their differences. Preventive action can be instituted for all three diseases because of their vast similarities. The receptor binding and antibody neutralization proteins of these viruses can be further investigated and be used as protection against epidemic strains. The E2 and E1 viral proteins in Chikungunya have the same function as the E viral protein of Dengue [69]. Cases of all three of these diseases are underreported. The reason for this, especially for Zika infections, could be due to a cross-reaction between the different viruses, their overlapping clinical symptoms, immunological protection that is widespread in the local population, selflimiting nature and mild symptoms or asymptomatic nature of Zika, poor specific test for diagnosing, and poor system surveillance [39]. The three viruses can be transmitted through stem cell/bone marrow grafts, organs, and blood transfusions; hence, laboratory confirmation using RT-PCR is required; however, DENV and ZIKV may cross-react [70]. Widespread immunologic protection by herd immunity in endemic countries perhaps leads to underreporting of cases, as well as providing grounds for uninfected countries to be protected from the epidemics. The development of vaccines due to extensive research measures and a solid understanding of the viral proteins of each of these viruses is at different phases of clinical trials. These vaccines will be instrumental to the safe and long-lasting population immunity protection.

Vaccine evolution has proven to be a tedious process, and the race to its development may possibly be one of the most productive aspects, simply because the theory behind vaccination is to eradicate the diseases associated with these mosquito-borne vectors [71, 72]. The impact of Chikungunya, Dengue, and Zika viruses and their disease prevalence vary due to a variety of factors. Some countries, like Vietnam, are still struggling greatly with the burden of morbidity from Dengue, so much so, that it is considered a public health problem [73]. Researchers, from the city of Nha Trang in Vietnam, are carrying out surveillance on Dengue cases that have been notified by community health stations and public hospitals, whose findings demonstrate three critical features: (1) the city has a pronounced temporal and spatial heterogeneity in the incidence of Dengue; (2) children and young adults are most at risk of infections and disease; and (3) four viral serotypes have been reported within a single season $[73,74]$. Other researchers have reported on the global disease burden of the CHIKV and its spread across vast parts of the world, and how former and current cases of DENV outbreaks may provide insight into what to expect and how to approach the issues associated with these vector-borne illnesses $[16,75]$. Like the two former mosquito-borne viruses, ZIKV is the latest to have spread globally and is unlikely to be the last [76]. In fact, the presence of the Aedes mosquito vector during the period from 2015 to 2016 in New York City necessitates a wide response to the health department, in order to properly identify and respond to the local transmission of ZIKV, including sentinel surveillance and amplified mosquito control [77]. Hence, epidemiological assessment of the three diseases is mandatory in both endemic and non-endemic countries [70]. Given extensive international travel and commercial interplay, it is necessary to consider health in a global context with the ultimate goal of improving public health by strengthening global disease detection, the response to disease detection, the preventative measures, and the control strategies for eradicating Chikungunya, Dengue, and Zika viruses [78, 79].

Author Contributions Adekunle Sanyaolu: conception and design, approval of the final version, responsibility for accuracy, and integrity of all aspects of research; Oladapo Ayodele: data acquisition, analysis, and interpretation; Lorena Likaj: data acquisition, analysis, and interpretation; Aleksandra Marinkovic: project administration, data acquisition, analysis, and interpretation; Jennifer Locke: data acquisition, analysis, and interpretation; Miriam Ahmed: data acquisition, analysis, and interpretation; Odunayo Akanbi: data acquisition, analysis, and interpretation; Verner Orish: supervision and revising the article for intellectual content; Chuku Okorie: supervision and revising the article for intellectual content; Olanrewaju Badaru: design of methodology and approval of the final version.

\section{Compliance with Ethical Standards}

Conflict of Interest The authors declare that they have no conflict of interest.

Human and Animal Rights and Informed Consent This article does not contain any studies with human or animal subjects performed by any of the authors.

\section{References}

Papers of particular interest, published recently, have been highlighted as:

- Of importance

1. Epelboin Y, Talaga S, Epelboin L, Dusfour I. Zika virus: an updated review of competent or naturally infected mosquitoes. PLoS Negl Trop Dis. 2017;11(11):1-22. https://doi.org/10.1371/journal.pntd. 0005933.

2. Song BH, Yun SI, Woolley M, Lee YM. Zika virus: history, epidemiology, transmission, and clinical presentation. J Neuroimmunol. 2017 Jul 15;308:50-64.

3. Tharmarajah K, Mahalingam S, Zaid A. Chikungunya: vaccines and therapeutics. F1000Res. 2017;6:2114. 
4. Thiboutot MM, Kannan S, Kawalekar OU, Shedlock DJ, Khan AS, Sarangan G, et al. Chikungunya: a potentially emerging epidemic? PLoS Negl Trop Dis. 2010;4(4):e623.

5. Rodriguez-Roche R, Gould EA. Understanding the Dengue viruses and progress towards their control. Biomed Res Int. 2013:1-37. https://doi.org/10.1155/2013/690835.

6. Patterson J, Sammon M, Garg M. Dengue, Zika and Chikungunya: emerging arboviruses in the new world. West J Emerg Med. 2016;17(6):671. This article discusses the presentation and treatment of Dengue, Zika, and Chikungunya, as well as special challenges presented to the emergency physician in evaluating a patient with a suspected arbovirus infection.

7. Li R, Ding J, Ding G, Fan X, He Y, Wang X, et al. Zika virus infections: a review. Radiol Infect Dis. 2017;4(2):88-93.

8. Zannoli S, Morotti M, Denicolò A, Tassinari M, Chiesa C, Pierro A, et al. Global epidemiology of Zika and Chikungunya virus human infections. Microbiol Med. 2017;32(3).

9. Mohan A, Kiran DH, Manohar IC, Kumar DP. Epidemiology, clinical manifestations, and diagnosis of Chikungunya fever: lessons learned from the re-emerging epidemic. Indian J Dermatol. 2010;55(1):54-63.

10. Caglioti C, Bordi L, Lalle E, Castilletti C, Capobianchi MR. Chikungunya virus infection: an overview. New Microbiol. 2013;36(1):211-27 http://www.newmicrobiologica.org/PUB/ allegati_pdf/2013/3/211.pdf. Accessed 15 Feb 2018.

11. Staples JE, Breiman RF, Powers AM. Chikungunya fever: an epidemiological review of a re-emerging infectious disease. Clin Infect Dis. 2009 Sep 15;49(6):942-8. https://doi.org/10.1086/605496.

12. Ganesan V, Duan B, Reid S. Chikungunya virus: pathophysiology, mechanism, and modeling. Viruses. 2017;9(12):368.

13. de Lamballerie X, Leroy E, Charrel RN, Ttsetsarkin K, Higgs S, Gould EA. Chikungunya virus adapts to tiger mosquito via evolutionary convergence: a sign of things to come? Virol J. 2008;5(1): 33. https://doi.org/10.1186/1743-422X-5-33.

14. Coffey LL, Failloux A-B, Weaver SC. Chikungunya virus-vector interactions. Viruses. 2014;6(11):4628-63. https://doi.org/10.3390/ v6114628.

15. Centers for Disease Control and Prevention (CDC). 2014 final data for the United States | chikungunya virus; 2015. Retrieved from https://www.cdc.gov/chikungunya/geo/united-states-2014.html on January 22, 2018.

16. Sanyaolu A, Okorie C, Badaru O, Wynveen E, White S, Wallace W. Chikungunya epidemiology: a global perspective. SM J Publ Health Epidemiol. 2016;2:1028.

17. Schwameis M, Buchtele N, Wadowski PP, Schoergenhofer C, Jilma B. Chikungunya vaccines in development. Hum Vaccin Immunother. 2016;12(3):716-31. https://doi.org/10.1080/ 21645515.2015.1101197.

18. National Institute of Allergy and Infectious Diseases (NIAID). NIAID - sponsored trial of experimental chikungunya vaccine begins; 2017. Retrieved from https://www.nih.gov/news-events/ news-releases/niaid-sponsored-trial-experimental-chikungunyavaccine-begins on January 22, 2018.

19. Shepard DS, Coudeville L, Halasa YA, Zambrano B, Dayan GH. Economic impact of Dengue illness in the Americas. Am J Trop Med Hyg. 2011;84(2):200-7.

20. Benelli, Giovanni, Mehlhorn H. Declining malaria, rising of Dengue and Zika virus: insights for mosquito vector control. Parasitol Res. 2016;115:1747-54.

21. Carabali M, Hernandez LM, Arauz MJ, Villar LA, Ridde V. Why are people with dengue dying? A scoping review of determinants for dengue mortality. BMC Infect Dis. 2015;15(301):1-22. https:// doi.org/10.1186/s12879-015-1058-x.

22. Simmons CP, Farrar J. Changing patterns of Dengue epidemiology and implications for clinical management and vaccines. PLoS Med. 2009;6(9):1-2.
23. Warkentien T, Pavlicek R. Dengue fever: historical perspective and the global response. J Infect Dis Epidemiol. 2016;2(2):1-6 https:/ www.clinmedjournals.org/articles/jide/journal-of-infectiousdiseases-and-epidemiology-jide-2-015.pdf. Accessed 15 Feb 2018.

24. San Martín JL, Brathwaite O, Zambrano B, Solórzano JO, Bouckenooghe A, Dayan GH, et al. The epidemiology of Dengue in the Americas over the last three decades: a worrisome reality. Am Soc Trop Med Hyg. 2010;82(1):128-35.

25. Kraemer MU, Sinka ME, Duda KA, Mylne AQ, Shearer FM, Barker CM, et al. The global distribution of the arbovirus vectors Aedes aegypti and Ae. albopictus. elife. 2015;4:e08347.

26. Liu LE, Dehning M, Phipps A, Swienton RE, Harris CA, Klein KR. Clinical update on dengue, chikungunya, and zika: what we know at the time of article submission. Disaster Med Public Health Prep. 2017;11(3):290-9. This article reviews the recent literature on the Zika virus as well as Chikungunya virus and Dengue virus. The article findings give a better understanding of the similarities and differences between the 3 infections in terms of their characteristics, clinical presentation, diagnosis methodology, and treatment and what can be done for prevention.

27. Rezza G. Dengue and Chikungunya: long-distance spread and outbreaks in naïve areas. Pathog Glob Health. 2014;108(8):349-55. https://doi.org/10.1179/2047773214Y.0000000163.

28. Lee VJ, Chow A, Zheng X, Carrasco LR, Cook AR, Lye DC, et al. Simple clinical and laboratory predictors of Chikungunya versus dengue infections in adults. PLoS Negl Trop Dis. 2012;6(9): e1786.1-12. https://doi.org/10.1371/journal.pntd.0001786.

29. Sanyaolu A, Okorie C, Badaru O, Adetona K, Ahmed M, Akanbi O, Foncham J, Kadavil S, Likaj L, Miraaj-Raza S, Pearce E, Sylvester R, Wallis E. Global epidemiology of dengue hemorrhagic fever: an update. Review Article. 2017;5(6):00179. https://doi.org/ 10.15406/jhvrv.2017.05.00179.

30. Pal S, Dauner AL, Valks A, Forshey BM, Long KC, Thaisomboonsuk B, et al. Multicountry prospective clinical evaluation of two enzyme-linked immunosorbent assays and two rapid diagnostic tests for diagnosing dengue fever. J Clin Microbiol. 2015;53(4):1092-102.

31. Goncalves de Castro M, Bruycker de Nogueira F, Ribeiro Nogueira RM, Lourenco-de-Oliveira R, Barreto dos Santos F. Genetic variation in the $3^{\prime}$ untranslated region of dengue virus serotype 3 strains isolated from mosquitoes and humans in Brazil. Virol J. 2013;10(3):1-11.

32. LaDeau SL, Allan BF, Leisnham PT, Levy MZ. Ecology of organisms in urban environments: the ecological foundations of transmission potential and vector-borne disease in urban landscapes. Funct Ecol. 2015;29:889-901.

33. Hause AM, Perez-Padilla J, Horiuchi K, Han GS, Hunsperger E, Aiwazian J, et al. Epidemiology of dengue among children aged < 18 months-Puerto Rico, 1999-2011. Am J Trop Med Hyg. 2016;94(2):404-8.

34. Martina BEE, Koraka P, Osterhaus ADME. Dengue virus pathogenesis: an integrated view. Clin Microbiol Rev. 2009;22(4):564-81.

35. World Health Organization. Chapter 2: clinical diagnosis. In: Dengue: guidelines for diagnosis, treatment, prevention, and control. Geneva: TDR; 2009. p. 12-23.

36. Jeewandara C, Adikari TN, Gomes L, Fernando S, Fernando RH, et al. Functionality of Dengue virus specific memory $\mathrm{T}$ cell responses in individuals who were hospitalized or who had mild or subclinical Dengue infection. PLoS Negl Trop Dis. 2015;9(4):116. https://doi.org/10.1371/journal.pntd.0003673.

37. Flipse J, Smit JM. The complexity of a Dengue vaccine: a review of the human antibody response. PLoS Negl Trop Dis. 2015;9(6):118. https://doi.org/10.1371/journal.pntd.0003749.

38. McArthur MA, Sztein MB, Edelman R. Dengue vaccines: recent developments, ongoing challenges and current candidates. Expert Rev Vaccines. 2013;12(8):933-53 https://www.ncbi.nlm.nih.gov/ pubmed/23984962. Accessed 15 Feb 2018. 
39. Kindhauser MK, Allen T, Frank V, Santhana RS, Dye C. Zika: the origin and spread of a mosquito-borne virus. Bull World Health Organ. 2016;94(9):675-686C. https://doi.org/10.2471/BLT.16. 171082.

40. Saiz J-C, Martín-Acebes MA, Bueno-Marí R, Salomón OD, Villamil-Jiménez LC, Heukelbach J, et al. Zika virus: what have we learnt since the start of the recent epidemic? Frontiers in Microbiology. 2017;8:1554-79 https://www.frontiersin.org/article/ 10.3389/fmicb.2017.01554. Accessed 15 Feb 2018.

41. Marano G, Pupella S, Vaglio S, Liumbruno GM, Grazzini G. Zika virus and the never-ending story of emerging pathogens and transfusion medicine. J Blood Transfus. 2016;14:95-100.

42. Fellner C. Zika virus: anatomy of a global health crisis. P\&T. 2016;41(4):242-53.

43. White MK, Wollebo HS, Beckham JD, Tyler KL, Khalili K. Zika virus: an emergent neuropathological agent. Ann Neurol. 2016;80(4):479-89. https://doi.org/10.1002/ana.24748.

44. Mansuy J, Mengelle C, Pasquier C, Chapuy-Regaud S, Delobel P, Martin-Blondel $\mathrm{G}$, et al. Zika virus infection and prolonged viremia in whole-blood specimens. Emerg Infect Dis. 2017;23(5):863-5. https://doi.org/10.3201/eid2305.161631.

45. Ali A, Wahid B, Rafique S, Idrees M. Advances in research on Zika virus. Asian Pac J Trop Med. 2017;10(4):321-31 https:/ac.els-cdn. com/S1995764517301682/1-s2.0-S1995764517301682-main. pdf? tid =spdf-dd5a 0 c $79-0$ bd $7-4609-8$ c 22 e $57253 \mathrm{fc}$ e $34 \mathrm{~d} \&$ a c d nat $=1519682727$ 91305c268b7e7fbf3df3ba4c98908222. Accessed 15 Feb 2018.

46. Musso D, Gubler DJ. Zika virus. Clin Microbiol Rev. 2016;29(3): 487-524. https://doi.org/10.1128/CMR.00072-15.

47. Furuya $\mathrm{H}$. Estimating vector-borne viral infections in the urban setting of the 2020 Tokyo Olympics, Japan, using mathematical modeling. Tokai J Exp Clin Med. 2017;42(4):160-4.

48. Pan American Health Organization/World Health Organization. Epidemiological update: Zika virus infection; 2015, 1-7. Retrieved from http://www.paho.org/hq/index.php?option=com docman \&task $=$ doc_view\&Itemid $=270 \&$ gid $=32021 \& l a n g=e n$ on Febuary 28, 2018

49. Faria NR, da Silva Azevedo RD, Kraemer MU, Souza R, Cunha MS, Hill SC, et al. Zika virus in the Americas: early epidemiological and genetic findings. Science. 2016;352(6283):345-9.

50. Foy BD, Kobylinski KC, Chilson Foy JL, Blitvich BJ, Travassos da Rosa A, Haddow AD, et al. Probable non-vector-borne transmission of Zika virus, Colorado, USA. Emerg Infect Dis. 2011;17(5): 880-2 https://www.ncbi.nlm.nih.gov/pmc/articles/PMC3321795/. Accessed 15 Feb 2018.

51. Musso D, Cao-Lormeau, Mai V, Gubler DJ. Zika virus: following the path of dengue and chikungunya? Lancet. 2015;386:243-4. On May 7, 2015, the Pan American Health Organization issued an alert about potential Zika virus (ZIKV) transmission in northeast Brazil. This was confirmed with wide spread of the disease, underscoring the potential for ZIKV to spread globally, similar to Dengue (DENV) and Chikungunya (CHIKV) viruses.

52. Rozé B, Najioullah F, Signate A, Apetse K, Brouste Y, Gourgoudou $\mathrm{S}$, et al. Zika virus detection in cerebrospinal fluid from two patients with encephalopathy, Martinique, February 2016. Eurosurveillance. 2016;21(16) https://www.ncbi.nlm.nih.gov/pubmed/27123558. Accessed 15 Feb 2018.

53. Cao-Lormeau VM, Blake A, Mons S, Lastère S, Roche C, Vanhomwegen J, et al. Guillain-Barré syndrome outbreak associated with Zika virus infection in French Polynesia: a case-control study. Lancet. 2016;387(10027):1531-9. https://doi.org/10.1016/ S0140-6736(16)00562-6.

54. Brasil P, Pereira JP, Moreira ME, Nogueira RMR, Damasceno L, Wakimoto M, et al. Zika virus infection in pregnant women in Rio de Janeiro. N Engl J Med. 2016;375(24):2321-34. https://doi.org/ 10.1056/NEJMoa1602412.
55. Soares de Oliveira-Szejnfeld P, Levine D, Melo AS, Amorim MM, Batista AG, Chimelli L, et al. Congenital brain abnormalities and Zika virus: what the radiologist can expect to see prenatally and postnatally. Radiology. 2016;281(1):203-18 https://www.ncbi.nlm. nih.gov/pubmed/27552432. Accessed 15 Feb 2018.

56. Ventura CV, Maja M, Bravo-Filho V, Gois AL, Belfort R Jr. Zika virus in Brazil and macular atrophy in a child with microcephaly. Lancet. 2016;387(10015):228 http://www.thelancet.com/journals/ lancet/article/PIIS0140-6736(16)00006-4/fulltext. Accessed 15 Feb 2018.

57. Martín-Acebes MA, Saiz J-C. West Nile virus: a re-emerging pathogen revisited. World J Virol. 2012;1(2):51-70 https://www.ncbi. nlm.nih.gov/pmc/articles/PMC3782267/. Accessed 15 Feb 2018.

58. World Health Organization. WHO vaccine pipeline tracker; 2017. Retrieved from http://www.who.int/immunization/research/vaccine pipeline_tracker_spreadsheet/en/retrieved on March 4, 2018.

59. Malone RW, Homan J, Callahan MV, Glasspool-Malone J, Damodaran L, et al. Zika virus: medical countermeasure development challenges. PLoS Negl Trop Dis. 2016;10(3):1-28. https:// doi.org/10.1371/journal.pntd.0004530.

60. Ioos S, Mallet HP, Leparc Goffart I, Gauthier V, Cardoso T, Herida M. Current Zika virus epidemiology and recent epidemics. Med Mal Infect. 2014;44(7):302-7 https://www.ncbi.nlm.nih.gov/ pubmed/25001879. Accessed 15 Feb 2018.

61. Davidson A, Slavinski S, Komoto K, Rakeman J, Weiss D. Suspected female-to-male sexual transmission of Zika virusNew York City. Morb Mortal Wkly Rep. 2016;65(28):716-7 https://www.cdc.gov/mmwr/volumes/65/wr/mm6528e2.htm. Accessed 15 Feb 2018.

62. Ciota AT, Bialosuknia SM, Ehrbar DJ, Kramer LD. Vertical transmission of Zika virus by Aedes aegypti and Ae. albopictus mosquitoes. Emerg Infect Dis. 2017;23(5):880-2. https://doi.org/10.3201/ eid2305.162041.

63. Charrel RN, Leparc-Goffart I, Pas S, de Lamballerie X, Koopmans $\mathrm{M}$, Reusken C. Background review for diagnostic test development for Zika virus infection. Bull World Health Organ. 2016;94(8):574 584D. https://doi.org/10.2471/BLT.16.171207.

64. Shapshak P, Wills T, Sinnott JT, Somboonwit C, Kuhn J, et al., editors. Global virology I-identifying and investigating viral diseases. 1st ed. New York: Springer-Verlag New York; 2015. p. 477500.

65. Figueiredo ML, Figueiredo LT. Emerging alphaviruses in the Americas: Chikungunya and Mayaro. Rev Soc Bras Med Trop. 2014;47(6):677-83 http://portal.crfsp.org.br/documentos/dengue/ artigos/Chikungunya/Emerging\%20alphaviruses\%20in\%20the\% 20Americas-\%20Chikungunya\%20and\%20Mayaro.pdf. Accessed $15 \mathrm{Feb} 2018$.

66. Haddow AD, Schuh AJ, Yasuda CY, Kasper MR, Heang V, Huy R, et al. Genetic characterization of Zika virus strains: Geographic expansion of the Asian lineage. PLoS Negl Trop Dis. 2012;6(2): e1477 http://journals.plos.org/plosntds/article?id=10.1371/journal. pntd.0001477. Accessed 15 Feb 2018.

67. Petersen EE, Polen KN, Meaney-Delman D, Ellington SR, Oduyebo T, Cohn A, et al. Update: interim guidance for health care providers caring for women of reproductive age with possible Zika virus exposure - United States, 2016. Morb Mortal Wkly Rep. 2016;65(12):315-22 https://www.cdc.gov/mmwr/volumes/65/wr/ mm6512e2.htm.

68. Heilman JM, De Wolff J, Beards GM, Basden BJ. Dengue fever: a Wikipedia clinical review. Open Med. 2014;8(4):105-15 https:// www.ncbi.nlm.nih.gov/pubmed/25426178. Accessed 15 Feb 2018.

69. Lo Presti A, Lai A, Cella E, Zehender G, Ciccozzi M. Chikungunya virus, epidemiology, clinics and phylogenesis: a review. Asian Pac J Trop Med. 2014;7(12):925-32 https://www.sciencedirect.com/ science/article/pii/S1995764514601644. Accessed 15 Feb 2018. 
70. Darrigo LG, Carvalho AM, Machado CM. Chikungunya, dengue, and Zika in immunocompromised hosts. Curr Infect Dis Rep. 2018;20(4):5.

71. Shragai T, Tesla B, Murdock C, Harrington LC. Zika and Chikungunya: mosquito-borne viruses in a changing world. Ann N Y Acad Sci. 2017;1399:61-77.

72. Obenauer JF, Joyner TA, Harris JB. The importance of human population characteristics in modeling Aedes aegypti distributions and assessing risk of mosquito-borne infectious diseases. Trop Med Health. 2017;45(38):1-9 http://tropmedhealth.biomedcentral.com/ articles/10.1186/s41182-017-0078-1. Accessed 15 Feb 2018.

73. Le Quyen D, Le NT, Van Anh CT, Nguyen NB, Van Hoang D, Montgomery JL, et al. Epidemiological, serological, and virological features of Dengue in Nha Trang City, Vietnam. Am J Trop Med Hyg. 2018;98(2):402-9. https://doi.org/10.4269/ajtmh.17-0630.

74. Hadinegoro SR, Arredondo-García JL, Capeding MR, Deseda C, Chotpitayasunondh T, Dietze R, et al. Efficacy and long-term safety of a dengue vaccine in regions of endemic disease. N Engl J Med. 2015;373(13):1195-206.

75. Shepard DS, Undurraga EA, Betancourt-Cravioto M, Guzmán MG, Halstead SB, Harris E, et al. Approaches to refining estimates of global burden and economics of dengue. PLoS Negl Trop Dis. 2014 Nov 20;8(11):e3306. https://doi.org/10.1371/journal.pntd. 0003306
76. Vythilingam I. Zika virus and its potential re-emergence in Malaysia. Med J Malaysia. 2016;71:68-70.

77. Lee CT, Vora NM, Bajwa W, et al. Zika Virus surveillance and preparedness-New York City, 2015-2016. MMWR Morb Mortal Wkly Rep. 2016;65:629-35. https://doi.org/10.15585/ mmwr.mm6524e3.

78. Powers AM, Waterman SH. A decade of arboviral activitylessons learned from the trenches. PLoS Negl Trop Dis. 2017;11(4):1-4 http://journals.plos.org/plosntds/article?id=10. 1371/journal.pntd.0005421. Accessed 15 Feb 2018.

79. Cara E. Vaccines for Zika, Chikungunya, Dengue update: the latest news from the lab; 2016. Retrieved from http://www.medicaldaily. com/vaccines-zika-chikungunya-dengue-update-latest-news-lab396992 on January 22, 2018.

Publisher's Note Springer Nature remains neutral with regard to jurisdictional claims in published maps and institutional affiliations. 